\title{
Devil's Vortex Phase Structure as Frequency Plane Mask for Image Encryption Using the Fractional Mellin Transform
}

\author{
Sunanda Vashisth, Hukum Singh, A. K. Yadav, and Kehar Singh \\ Department of Applied Science, ITM University, Sector 23-A, Gurgaon, Haryana 122 017, India \\ Correspondence should be addressed to Sunanda Vashisth; sunandavashisht@itmindia.edu
}

Received 19 February 2014; Revised 14 April 2014; Accepted 14 April 2014; Published 7 May 2014

Academic Editor: Yu S. Kivshar

Copyright (C) 2014 Sunanda Vashisth et al. This is an open access article distributed under the Creative Commons Attribution License, which permits unrestricted use, distribution, and reproduction in any medium, provided the original work is properly cited.

\begin{abstract}
A frequency plane phase mask based on Devil's vortex structure has been used for image encryption using the fractional Mellin transform. The phase key for decryption is obtained by an iterative phase retrieval algorithm. The proposed scheme has been validated for grayscale secret target images, by numerical simulation. The efficacy of the scheme has been evaluated by computing mean-squared-error between the secret target image and the decrypted image. Sensitivity analysis of the decryption process to variations in various encryption parameters has been carried out. The proposed encryption scheme has been seen to exhibit reasonable robustness against occlusion attack.
\end{abstract}

\section{Introduction}

Phase filters play an important role in designing an optical image encryption system. One of the most efficient zone plates is based on Devil's vortex Fresnel lens (DVFL) derived from Devil's staircase function called the Cantor function [1]. This function, related to the standard Cantor set, finds applications in areas like wave-particle interactions [2], crystal growth [3], and mode locking of the 3D coherent states in high-Q laser cavities [4]. Devil's staircase also shows up in the context $[5,6]$ of dynamical systems and periodic structures with long spatial periods and so forth. Many such examples along with their physical interpretation are given in [5-8]. Devil's lens (DL), also called blazed fractal zone plate, has a phase distribution much similar to a ramped stair-step pattern and has a quadratic fractal blazed profile $[9,10]$. Devil's vortex lens (DVL) is phase-only Devil's lens modulated by a helical structure [11-14]. When a spiral phase plate is introduced, the DVL generates multiple axial vortices. When a converging Fresnel lens is incorporated in the DVL, it results in a DVFL [15]. Barrera et al. [16] have presented results on multiple image encryptions and multiplexing based on fractal encrypting masks, using joint transform correlator architecture. In the present study, we have used DVFL as a structured phase filter in the frequency domain of a fractional Mellin transform-based encryption system to attain enhanced security.

A number of image encryption studies carried out in the past are based on the double random phase encoding (DRPE) technique. However, DRPE using the Fourier transform (FT) suffers from the problems of security and its vulnerability to a variety of attacks [17]. This has motivated researchers to design more secure systems by introducing additional parameters through phase filters and the use of a variety of integral transforms such as the fractional Fourier transform $[18,19]$, gyrator transform $[20,21]$, the Hartley transform $[22,23]$, and the Arnold transform [24]. It is now well known that optical and hybrid techniques for information security [25-27] have several advantages over the digital techniques.

The encryption scheme proposed in our paper is asymmetric and is based on the FrMT [28-30]. Compared to most transform-based techniques which are linear in nature, the fractional Mellin transform (FrMT) is a nonlinear transform and could potentially provide security against most known attacks [17, 31]. Here, we have used FrMT on an arbitrary input image transformed to an annular domain. The transformed image is multiplied by a structured phase mask (DVFL) in the frequency domain, aimed at enhancing the 
TABLE 1: Values of $q_{s, l}$ and $p_{s, l+1}$ for $S=0,1,2$, and 3 [15].

\begin{tabular}{lcll}
\hline S. Number & Values of $S$ & $q_{s, l}$ and $p_{s, l+1}$ & Values of $q_{s, l}$ and $p_{s, l+1}$ \\
\hline 1. & $S=0$ & $q_{0,0} p_{0,1}$ & 0,1 \\
2. & $S=1$ & $q_{1,0} p_{1,1} q_{1,1} p_{1,2}$ & $0,1 / 3,2 / 3,1$ \\
3. & $S=2$ & $q_{2,0}, p_{2,1}, q_{2,1}, p_{2,2} q_{2,2} p_{2,3} q_{2,3} p_{2,4}$ & $0,1 / 9,2 / 9,1 / 3,2 / 3,7 / 9,8 / 9,1$ \\
& & $q_{3,0}, p_{3,1}, q_{3,1}, p_{3,2} q_{3,2} p_{3,3} q_{3,3} p_{3,4}$, & $0,1 / 27,2 / 27,3 / 27,6 / 27,7 / 27,8 / 27,9 / 27,18 / 27$, \\
& $S=3$ & $q_{3,4} p_{3,5}, q_{3,5} p_{3,6}, q_{3,6} p_{3,7}, q_{3,7} p_{3,8}$ & $19 / 27,20 / 27,21 / 27,24 / 27,25 / 27,26 / 27,1$ \\
\hline
\end{tabular}

security by increasing the key space. This is followed by phase retrieval algorithm (PRA) $[32,33]$ to generate the phase key for decryption.

The paper is organised as follows. The construction of phase function based on DVFL is explained in Section 2. The details of the encryption-decryption processes and PRA are given in Section 3. In Section 4, we have presented the results of numerical simulation followed by conclusions in Section 5 .

\section{DVFL-Based Phase Function}

A phase mask based on Devil's lens can be described by the one-dimensional Cantor function, a particular case of Devil's staircase. A triadic Cantor set in the interval $[0,1]$ can be defined as [9]

$$
F_{S}(x)= \begin{cases}\frac{l}{2^{S}} & \text { if } p_{S, l} \leq x \leq q_{S, l}, \\ \frac{1}{2^{S}} \frac{x-q_{S, l}}{p_{S, l+1}-q_{S, l}}+\frac{l}{2^{S}} & \text { if } q_{S, l} \leq x \leq p_{S, l+1},\end{cases}
$$

where $F_{S}(0)=0, F_{S}(1)=1, S$ is the order of the Cantor function, and $l$ defines the number of horizontal sections of the function, having a value from 0 to $2^{S}-1$. Here $q$ and $p$ are the start and end points of each segment of the Cantor set. For some basic values of $S$, the values of $q_{S, l}$ and $p_{S, l+1}$ are provided in Table 1 [15].

From (1), one can easily note that the function $F_{S}(x)$ increases linearly from $q_{S, l}$ to $p_{S, l+1}$. The phase function corresponding to Devil's lens is defined as

$$
q(\varsigma)=q_{\mathrm{DL}}(\varsigma, S)=\exp \left[-i 2^{S+1} \pi F_{S}(\varsigma)\right],
$$

where $\varsigma=(r / a)^{2}$ is the normalized quadratic radial coordinate and $a$ is the lens radius. The phase function $q(\varsigma)$ increases linearly from 0 to $2 \pi$. A DVL can be constructed by combining Devil's lens and a vortex lens (VL). A vortex lens is based on an azimuthally dependent vortex function, and its phase function can be given as $V_{m}(\theta)=\exp (\operatorname{im} \theta)$, where $m$ is a nonzero integer called topological charge and $\theta$ is the azimuthal angle. Incidentally, the concept of topological charge has given rise to an important branch of optics called singular optics which plays an important role in various contexts (e.g., $[34,35])$.

The phase function corresponding to DVL can be given as

$$
\begin{gathered}
\Phi_{\mathrm{DVL}}(\zeta, \theta)=q(\zeta) \times V_{m}(\theta) \\
\Phi_{\mathrm{DVL}}(\zeta, \theta)=\exp \left[-i 2^{s+1} \pi F_{s}(\zeta)\right] \exp (i m \theta)
\end{gathered}
$$

In the same manner, we can combine a Fresnel zone plate with DVL. The phase function of a Fresnel zone plate is defined as [15]

$$
L_{f_{0}}(\zeta)=\exp \left[-i \pi \zeta \frac{a^{2}}{\lambda f_{0}}\right]
$$

where $f_{0}$ is focal length and $\lambda$ is the incident wavelength. A combination of the Fresnel lens (FL) and DVL results in the formation of DVFL, having a phase function given by [15]

$$
\begin{aligned}
\Omega_{S, m, f_{0}}(\zeta, \theta) & =q(\zeta) \times V_{m}(\theta) \times L_{f_{0}}(\zeta), \\
\Omega_{S, m, f_{0}}(\zeta, \theta)= & \exp \left[-i 2^{s+1} \pi F_{s}(\zeta)\right] \\
& \times \exp (i m \theta) \exp \left[-i \pi \zeta \frac{a^{2}}{\lambda f_{0}}\right] .
\end{aligned}
$$

A plot of DVFL, which is a combination of Devil's lens, a vortex lens, and a Fresnel lens, is shown in Figure 1.

\section{The Encryption Scheme}

The scheme proposed in our paper is based on the FrMT, a nonlinear integral transform [28-30]. According to this, an arbitrary image is first transformed to log-polar coordinates and is then subjected to the fractional Fourier transform (FrFT), which is a generalization of the Fourier transform in fractional order [18] and provides additional degree of freedom for encryption.

The FrMT is inspired by the FrFT. A two-dimensional FrMT of order $\left(p_{1}, p_{2}\right)$ is the FrFT of the same order $\left(p_{1}, p_{2}\right)$ of a function in its log-polar transformation.

In a Cartesian coordinate system, the two-dimensional FrMT of order $\left(p_{1}, p_{2}\right)$ of an image $f(x, y)$ is given by [28]

$$
\begin{aligned}
& M^{p_{1}, p_{2}}(u, v) \\
& =\iint_{-\infty}^{+\infty} f(x, y) x^{-\left(2 i u \pi / \sin \Phi_{1}\right)-1} \\
& \quad \times \exp \left[\frac{i \pi\left(u^{2}+\ln ^{2} x\right)}{\tan \Phi_{1}}\right] \times y^{-\left(2 i v \pi / \sin \Phi_{2}\right)-1} \\
& \quad \times \exp \left[\frac{i \pi\left(v^{2}+\ln ^{2} y\right)}{\tan \Phi_{2}}\right] d x d y
\end{aligned}
$$



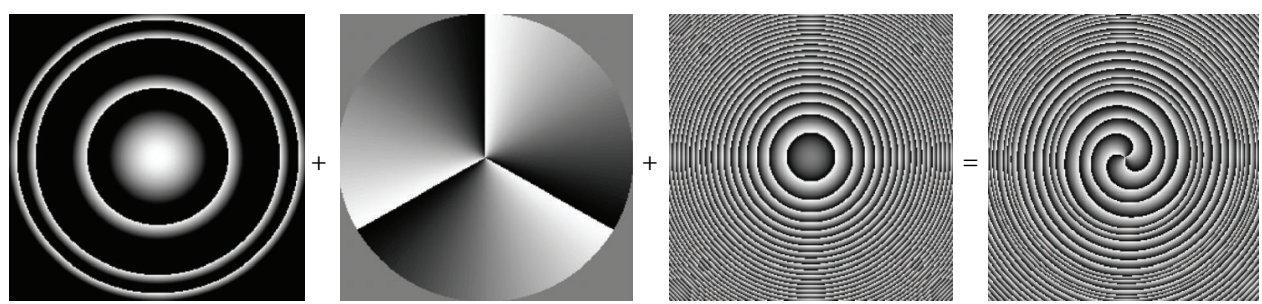

Figure 1: Phase plots of each component which when combined result in Devil's vortex Fresnel lens (DVFL). Sum of all components, that is, $S=2, m=3, \lambda=632.8 \mathrm{~nm}$, and $f=4 \mathrm{~cm}$, constitutes the final DVFL [15].

where $\Phi_{1}=p_{1} \pi / 2$ and $\Phi_{2}=p_{2} \pi / 2$. When the image is transformed to an annular domain by log-polar transformation, its FrMT of order $\left(p_{1}, p_{2}\right)$ can be written [28] as

$$
\begin{aligned}
& M^{p_{1}, p_{2}}(u, v) \\
& =C \iint_{-\infty}^{+\infty} f(\rho, \theta) \\
& \times \exp \left\lfloor-2 i \pi\left(\frac{u \rho}{\sin \Phi_{1}}+\frac{v \theta}{\sin \Phi_{2}}\right)\right. \\
& \left.\quad+\frac{i \pi\left(u^{2}+\rho^{2}\right)}{\tan \Phi_{1}}+\frac{i \pi\left(v^{2}+\theta^{2}\right)}{\tan \Phi_{2}}\right\rfloor d \rho d \theta \\
& =F^{p_{1}, p_{2}}\{f(\rho, \theta)\},
\end{aligned}
$$

where $C$ is a constant. Since FrMT involves log-polar transformation of the input image prior to its transformation by FrFT, it requires the setting up of parameters for transforming the input image to an annular domain. Hence, a few parameters are set in advance such as centre position of annular domain, $c_{x}, c_{y}$, the radii of the innermost $\left(r_{\text {in }}\right)$ and outermost $\left(r_{\text {out }}\right)$ rings of annular domain, and the number of sampling points along distance axis $n_{r}$ and along angle axis $n_{w}$.

Some recent image encryption studies have preferred structured phase masks over the commonly used random phase masks (RPM) because of some advantages. Unlike in the conventional DRPE scheme, here we have used a structured phase mask based on Devil's vortex Fresnel lens (DVFL) in the frequency plane [15] by multiplying the FrMT transformed image with phase function based on DVFL. This is followed by an iterative phase retrieval algorithm (PRA).

In the iterative process, the initial amplitude is taken from the FrMT transformed image and the initial phase is randomly generated with the uniform distribution in $[0,2 \pi]$ as in a recent study [30]. To initiate PRA, we take FrFT of order $\alpha$ of the initial input. The amplitude of the resulting complex function is replaced by a secret (target) amplitude image. Next, we perform the inverse FrFT to obtain complex distribution in the image plane. The phase of the transformed result substitutes the phase of input in the first step and the next iteration starts. The above steps are repeated until the algorithm converges or reaches a preset number of iterations.

We can summarize the steps of encryption scheme as follows. (i) Start with an arbitrary input image for generating ciphertext.

(ii) Transform the arbitrary image to log-polar coordinates and then subject it to a fractional Fourier transformation, resulting in a fractional Mellin transformed image.

(iii) Multiply the transformed image by a structured phase mask based on (DVFL).

(iv) Apply the PRA based on FrFT.

Flow chart of the encryption scheme including the PRA is shown in Figure 2. In our scheme, we consider an arbitrary real valued image $A(x, y)$. The selected annular domain image of $A(x, y)$ is $A_{r}(x, y)$ with inner and outer radii, respectively, as $r_{\text {in }}$ and $r_{\text {out }}$. The image in the annular domain is then subjected to FrFT of order $p$ (taken same in both directions, i.e., $p_{1}=p_{2}=p$ ). This transformed image is multiplied by a DVFL-based structured phase function. Thus, the transformed result $g(u, v)$, a complex-valued image, can be expressed as

$$
\begin{aligned}
g(u, v)= & A_{m}(u, v) \exp \left[i \phi_{0}(u, v)\right] \\
& \times \exp \left[i \phi_{1}(u, v)\right] \exp \left[i \phi_{2}(u, v)\right] \exp \left[i \phi_{3}(u, v)\right],
\end{aligned}
$$

where $\phi_{1}, \phi_{2}$, and $\phi_{3}$ are the phases of DL, VL, and FL, respectively, and $A_{m}(u, v)$ and $\phi_{0}$ are, respectively, the amplitude and phase of the FrMT transformed image.

The next step of the encryption process is the use of an iterative PRA to determine the phase key corresponding to the secret image [30]. The input amplitude of the complexvalued image for PRA can be written as

$$
A_{m}(u, v)=|g(u, v)| .
$$

The next step of PRA can be expressed mathematically as

$$
\begin{aligned}
& I(x, y) \exp (\operatorname{in}(x, y)) \\
& \quad=F^{\alpha_{1}, \alpha_{2}}\left\{A_{m}(u, v) \exp [i \theta(u, v)]\right\} .
\end{aligned}
$$

Initially $\theta(u, v)$ is taken as uniform random distribution in the interval $[0,2 \pi]$ and subsequently it is generated from the PRA iteratively. $F^{\alpha}$ on the right hand side of (10) represents the FrFT of order $\alpha$ (taken same in both directions, i.e., $\alpha_{1}=$ 


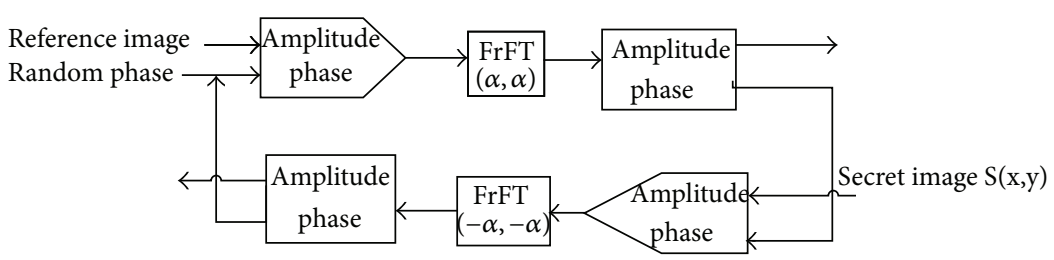

FIgURE 2: Flow chart of phase retrieval algorithm (adapted from Zhou et al. [30]).

$\left.\alpha_{2}=\alpha\right)$. Here, $n(x, y)$ is the phase distribution. The final step of the PRA can be written by taking the inverse FrFT as

$$
\begin{aligned}
& A^{\prime}(u, v) \exp \left(i \phi_{4}(u, v)\right) \\
& \quad=F^{-\alpha_{1},-\alpha_{2}}\{S(x, y) \exp [i n(x, y)]\},
\end{aligned}
$$

where $\phi_{4}(u, v)$ is the phase distribution obtained by iterative method and used in the first step. The phase key can be made complicated by modulating the phase functions, thereby enhancing the security of the system. The amplitude $A_{m}(u, v)$ of the transformed image is used to generate a phase distribution given by

$$
\phi_{5}(u, v)=2 \pi \frac{A_{m}(u, v)}{K} \in(0,2 \pi),
$$

where $K$ is a positive number equal to $1+\max \left(A_{m}\right)$.

The final phase key $\phi(u, v)$ is obtained from the following expression:

$$
\begin{aligned}
\phi(u, v)= & \phi_{4}(u, v)-\phi_{0}(u, v)-\phi_{1}(u, v) \\
& -\phi_{2}(u, v)-\phi_{3}(u, v)-\phi_{5}(u, v) .
\end{aligned}
$$

The keys of the proposed scheme include the order $\alpha$ of FrFT in the iterative process, the final phase key $\phi(u, v)$, and the order $p$ of the FrMT along with parameters of the structured phase mask, DVFL.

Decryption Process. Generally, the decryption process is reverse of the encryption. The transformed image $g(u, v)$ is multiplied by the final phase key obtained from (13). The resulting expression undergoes the fractional Fourier transformation of the order $\alpha$ to give complex distribution of the decrypted image. The amplitude distribution of the decrypted image is given by

$$
S(x, y)=\left|F^{\alpha_{1}, \alpha_{2}}\left[g(u, v) \exp \left\{i\left\lceil\phi(u, v)+\phi_{5}(u, v)\right]\right\}\right]\right| .
$$

\section{Numerical Simulations and Discussion}

The validity of the proposed scheme has been verified by performing some numerical simulations using MATLAB 7.6. An arbitrary input image Lena is chosen, which has $256 \times 256$ pixels and gray level is in the range of 0-255 (Figure 3(a)). To start the encryption process, some parameters have to be set since a selected annular domain of the ciphertext is transformed by FrMT. The FrFT in the scheme has been computed using an algorithm proposed by Garcia et al. [36].
For simplicity, the FrMT orders in both the directions are assumed to be the same and equal to 0.3 . The center position of the annular domain is taken as $\left(c_{x}, c_{y}\right)=(96,108)$, with the inner and outer radii being, respectively, $r_{\text {in }}=20, r_{\text {out }}=$ 80 [30]. The annular domain with these parameters has been shown in Figure 3(b), whereas the FrMT transformed amplitude image $A_{m}(u, v)$ is shown in Figure 3(c). The orders of the FrFT and IFrFT in the PRA are all taken equal to 0.7. The iterative process ends when the correlation coefficient (CC) value exceeds the predefined value 0.996 . Through PRA, we are able to obtain the corresponding decryption phase key which is the main key in the proposed scheme. When even one incorrect parameter of DVFL is used, one can hardly retrieve the original image with other correct parameters.

Using this scheme we have carried out numerical simulations using Lena as the arbitrary image and a secret image for encryption. As shown in Figure 4(a), the secret image chosen to be encrypted is a grayscale image of Barbara having a size of $256 \times 256$ pixels. The corresponding phase key generated from the PRA has been shown in Figure 4(b), and the decrypted image using the correct keys, that is, $(S=3, \lambda=632.8 \mathrm{~nm}, m=3, f=4 \mathrm{~cm}, p=0.3, \alpha=$ 0.7 ), is shown in Figure 4(c). Figures 5(a)-5(d) show the decrypted images with only one incorrect parameter (with all other parameters being correct). It is seen from Figure 5(d) that the decryption is not very sensitive to a change in the parameter $m$. Figure 5(e) shows the decrypted image with two incorrect parameters $S$ and $m$. The decrypted image is unrecognizable (except in Figure 5(d)), thus highlighting the scheme's sensitivity to the encryption parameters.

In order to assess the efficiency and security of the proposed scheme, the mean-squared-error (MSE) between the original image and the decrypted image has been calculated. If $I_{o}(x, y)$ and $I_{d}(x, y)$ denote, respectively, the pixel values of the original image and the decrypted image, the MSE function can be written as

$$
\operatorname{MSE}=\frac{1}{N \times N} \sum_{x=1}^{N} \sum_{y=1}^{N}\left|I_{o}(x, y)-I_{d}(x, y)\right|^{2} .
$$

Besides the decryption phase key obtained from the PRA, the proposed scheme also depends on some other factors such as the DVFL parameters, as well as orders of the FrMT and FrFT. In order to study the sensitivity of the encryption scheme to orders of the FrMT and FrFT, the MSE values have been plotted against the orders of the FrMT and FrFT in Figures 6(a) and 6(b), respectively. These results imply that the decryption of the original image is very sensitive to the orders of FrMT and FrFT. Based on the above numerical 


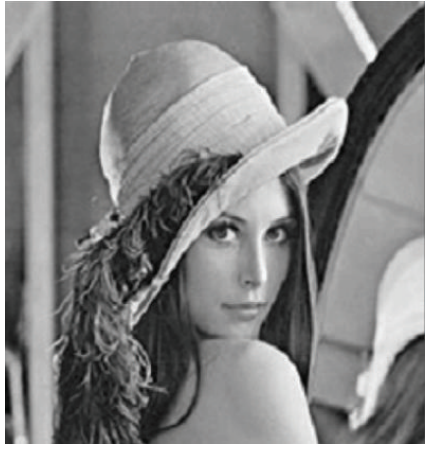

(a)

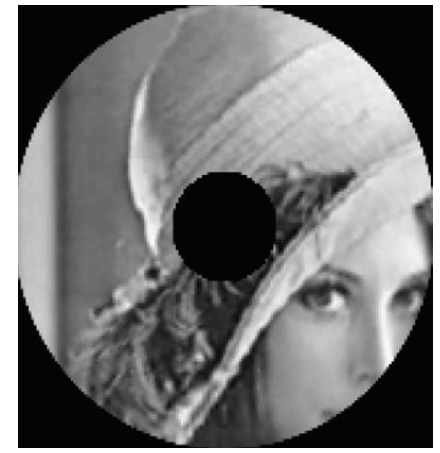

(b)

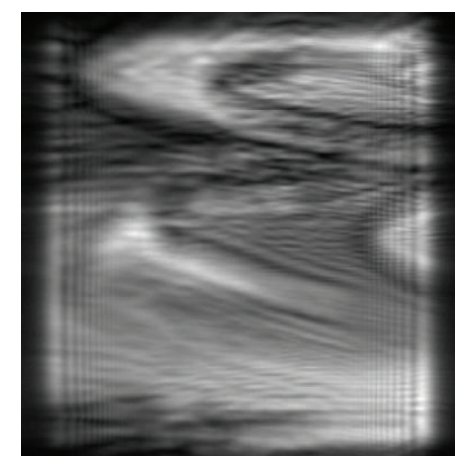

(c)

FiguRe 3: (a) Arbitrary Input grayscale image Lena with $256 \times 256$ pixels. (b) Annular domain image with center position $\left(c_{x}, c_{y}\right)=(96,108)$, and the inner and outer radii are $r_{\text {in }}=20, r_{\text {out }}=80$ [30]. (c) The fractional Mellin transformed amplitude image of order 0.3 in both directions.

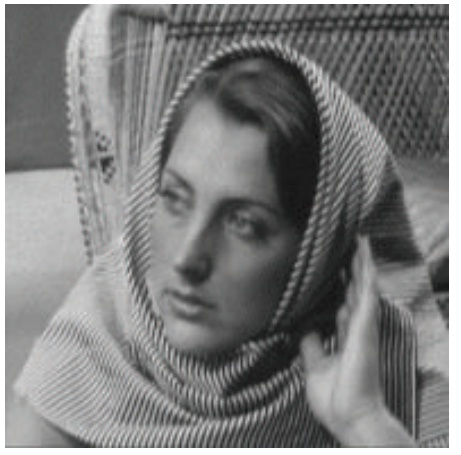

(a)

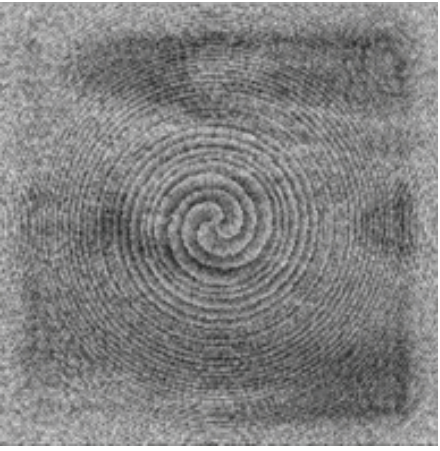

(b)

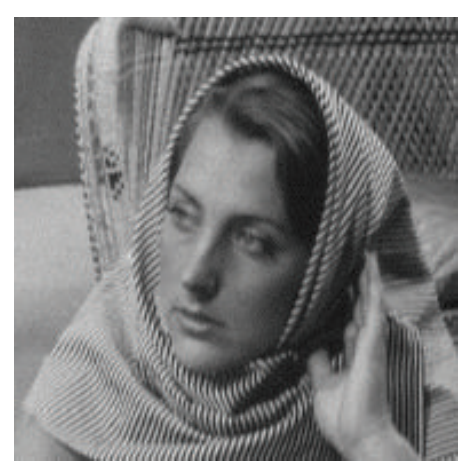

(c)

FIGURE 4: (a) Secret grayscale image with $256 \times 256$ pixels of Barbara, (b) the corresponding phase key generated by PRA, and (c) retrieved image with correct decryption parameters $(S=3, \lambda=632.8 \mathrm{~nm}, m=3, f=4 \mathrm{~cm}, p=0.3, \alpha=0.7)$. 


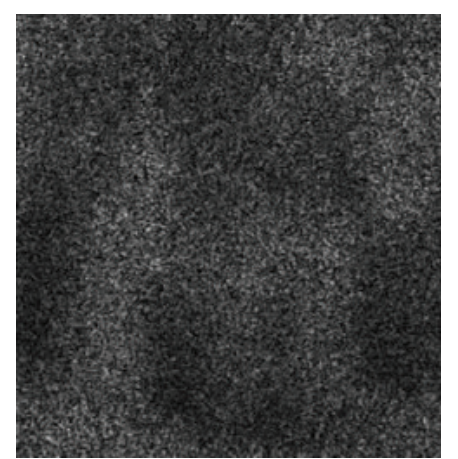

(a)

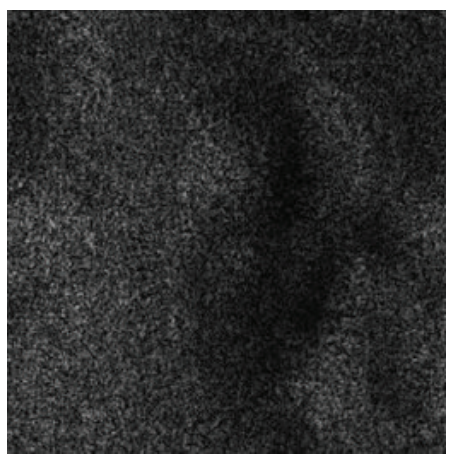

(c)

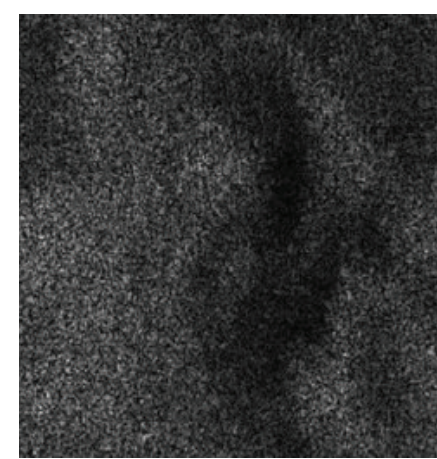

(b)

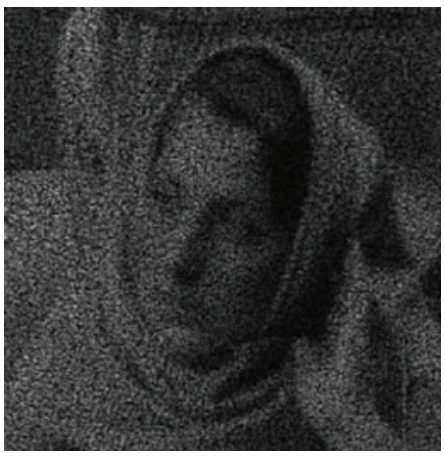

(d)

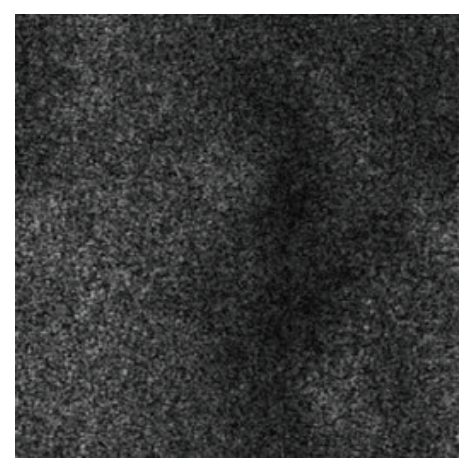

(e)

FIGURE 5: Decrypted image with one incorrect parameter, with others being correct. (a) $S=2$, (b) $\lambda=600 \mathrm{~nm}$, (c) $f=4.5 \mathrm{~cm}$, and (d) $m=1$. (e) Decrypted image with two incorrect parameters $(S=2, m=1)$.

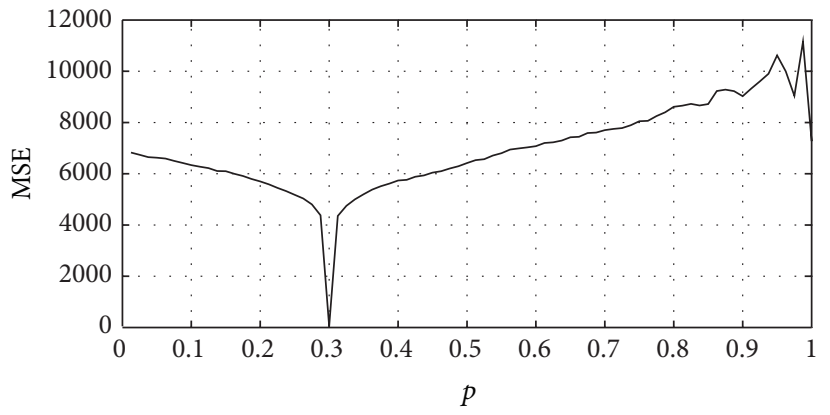

(a)

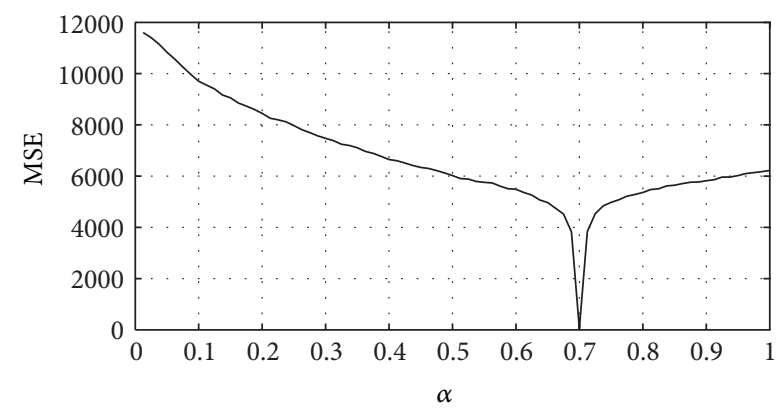

(b)

FIGURE 6: (a) MSE (between secret image and decrypted image) as a function of order of FrMT. (b) MSE (between secret image and decrypted image) as a function of order of FrFT. 


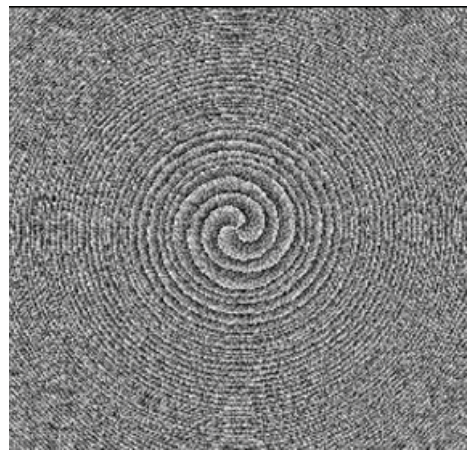

(a)

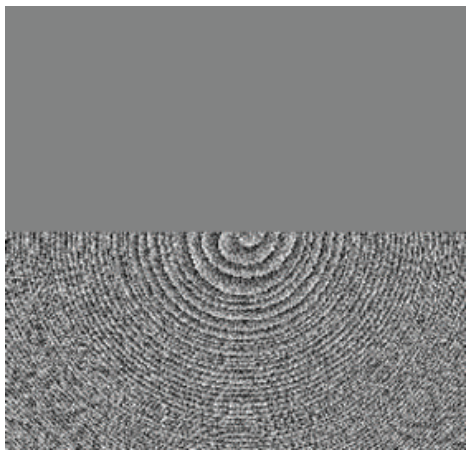

(c)

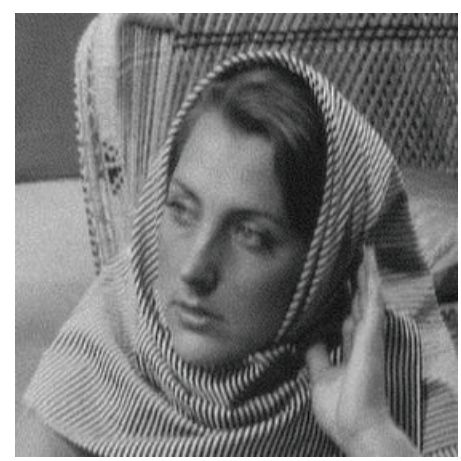

(e)

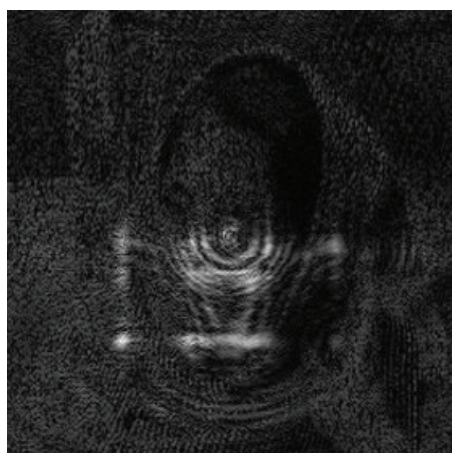

(g)

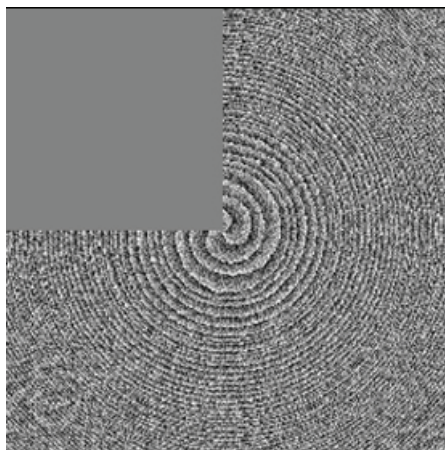

(b)

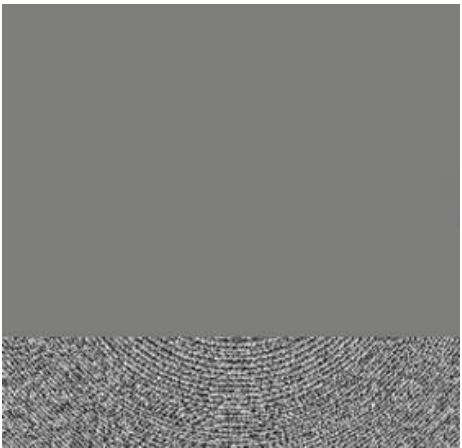

(d)

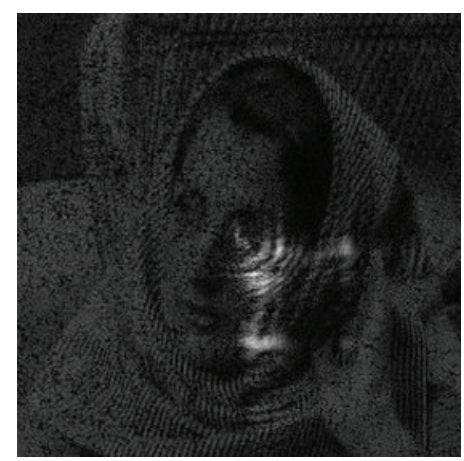

(f)

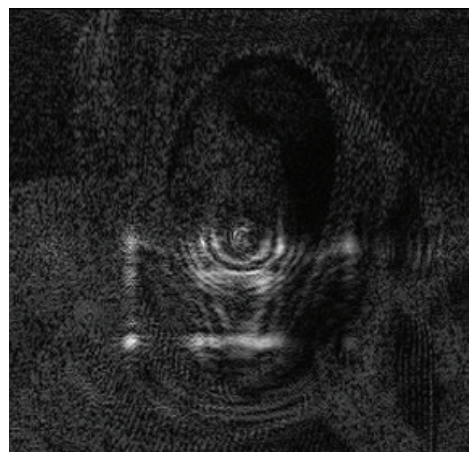

(h)

FIGURE 7: Impact of occlusion attack on decryption phase key. (a)Without occlusion, (b) $25 \%$ occlusion, (c) $50 \%$ occlusion, (d) $75 \%$ occlusion, and (e)-(h) corresponding retrieved images. 
simulations, one can see that all the correct keys are required in the decryption process. If any key of encryption process is incorrect, it becomes increasingly difficult to retrieve the secret image.

The robustness of the encryption scheme is tested against occlusion attacks. As the decryption key contains phase key generated by PRA, it has the phase information of the secret image. Thus, in this scheme, the phase key plays a vital role as it bears the signature of the secret image. We have subjected the decryption phase key to occlusion attacks as shown in Figure 7. The corresponding retrieved images shown alongside indicate reasonable robustness of the scheme up to $50 \%$ occlusion. It can be concluded that apparently the main information of the secret image can be recognized visually from the decrypted images.

\section{Conclusions}

An encryption scheme for grayscale image has been proposed, which uses a nonlinear fractional Mellin transform, a structured phase mask (Devil's vortex Fresnel lens) in the frequency plane, employing a phase retrieval technique to generate the decryption phase key corresponding to each secret input image. The use of FrMT with DVFL phase mask enlarges the key space, thereby making the proposed scheme more secure. The proposed scheme has been validated for a few grayscale images using MATLAB 7.6. The sensitivity of the scheme has also been studied for various parameters such as phase keys and orders of FrMT and FrFT. The scheme is considered secure against most of the known attacks by virtue of its design being nonlinear and asymmetric and having a larger key space. However, with the rapid increase in computational capability and emergence of new techniques, one can expect to break security of the system in the future. In this context, it may be worthwhile to carry out a cryptanalysis of the proposed scheme in the future with a view to investigating the endurance of the scheme against various other attacks.

\section{Conflict of Interests}

The authors declare that there is no conflict of interests regarding the publication of this paper.

\section{Acknowledgments}

The authors wish to thank Professor Prem Vrat and the management of the ITM University for their encouragement.

\section{References}

[1] D. R. Chalice, "A characterization of Cantor functions," The American Mathematical Monthly, vol. 98, pp. 255-258, 1991.

[2] F. Doveil, A. Macor, and Y. Elskens, "Direct observation of a "devil's staircase" in wave-particle interaction," Chaos, vol. 16, no. 3, Article ID 033103, 2006.

[3] M. Hupalo, J. Schamalian, and M. C. Tringides, "Devil's staircase in $\mathrm{Sb} / \mathrm{Si}(111)$ ordered phases," Physical Review Letters, vol. 90, Article ID 216106, 2003.
[4] Y. F. Chen, T. H. Lu, K. W. Su, and K. F. Huang, "Devil's staircase in three-dimensional coherent waves localized on Lissajous parametric surfaces," Physical Review Letters, vol. 96, no. 21, Article ID 213902, 2006.

[5] P. Bak, "Commensurate phases, incommensurate phases and the devil's staircase," Reports on Progress in Physics, vol. 45, no. 6, article 001, pp. 587-629, 1982.

[6] P. Bak, “The devil's staircase," Physics Today, pp. 38-45, 1986.

[7] P. Pieranski, P. Sotta, D. Rohe, and M. Imperor-Clerc, "Devil's staircase-type faceting of a cubic lyotropic liquid crystal," Physical Review Letters, vol. 84, no. 11, pp. 2409-2412, 2000.

[8] J. H. E. Cartwright, J. Douthett, D. L. González, R. Krantz, and O. Piro, "Two musical paths to the Farey series and devil's staircase," Journal of Mathematics and Music, vol. 4, no. 1, pp. 57-74, 2010.

[9] J. A. Monsoriu, W. D. Furlan, G. Saavedra, and F. Giménez, “Devil's lenses," Optics Express, vol. 15, no. 21, pp. 13858-13864, 2007.

[10] A. Calatayud, J. A. Monsoriu, O. Mendoza-Yero, and W. D. Furlan, "Polyadic devil's lenses," Journal of the Optical Society of America A, vol. 26, no. 12, pp. 2532-2537, 2009.

[11] W. D. Furlan, F. Giménez, A. Calatayud, and J. A. Monsoriu, "Devil's vortex-lenses," Optics Express, vol. 17, no. 24, pp. 2189121896, 2009.

[12] A. Calatayud, J. A. Rodrigo, L. Remón, W. D. Furlan, G. Cristóbal, and J. A. Monsoriu, "Experimental generation and characterization of Devil's vortex-lenses," Applied Physics B, vol. 106, no. 4, pp. 915-919, 2012.

[13] A. Calabuig, S. S. R. Lluis Martinez-Leon, E. Tajahuerce et al., "Generation of programmable 3D optical vortex structures through devil's vortex-lens arrays," Applied Optics, vol. 52, pp. 5822-5829, 2013.

[14] W. D. Furlan, F. Giménez, A. Calatayud, L. Remón, and J. A. Monsoriu, "Volumetric multiple optical traps produced by devil's lenses," Journal of the European Optical Society, vol. 5, Article ID 10037s, 2010.

[15] M. Mitry, D. C. Doughty, J. L. Chaloupka, and M. E. Anderson, "Experimental realization of the devil's vortex Fresnel lens with a programmable spatial light modulator," Applied Optics, vol. 51, pp. 4103-4108, 2012.

[16] J. F. Barrera, M. Tebaldi, D. Amaya et al., "Multiplexing of encrypted data using fractal masks," Optics Letters, vol. 37, pp. 2895-2897, 2012

[17] Y. Frauel, A. Castro, T. J. Naughton, and B. Javidi, "Resistance of the double random phase encryption against various attacks," Optics Express, vol. 15, no. 16, pp. 10253-10265, 2007.

[18] H. M. Ozaktas, Z. Zalevsky, and M. A. Kutay, Eds., The Fractional Fourier Transform with Applications in Optics and Signal Processing, John Wiley \& Sons, New York, NY, USA, 2000.

[19] G. Unnikrishnan and K. Singh, "Double random fractional Fourier-domain encoding for optical security," Optical Engineering, vol. 39, no. 11, pp. 2853-2859, 2000.

[20] J. A. Rodrigo, T. Alieva, and M. L. Calvo, "Gyrator transform: properties and applications," Optics Express, vol. 15, no. 5, pp. 2190-2203, 2007.

[21] Q. Wang, Q. Guo, and L. Lei, "Double image encryption based on phase-amplitude mixed encoding and multistage phase encoding in gyrator transform domains," Optics \& Laser Technology, vol. 48, pp. 267-279, 2013.

[22] Z.-j. Liu, M. A. Ahmad, and S.-t. Liu, "Image encryption based on double random amplitude coding in random hartley transform domain," Optik, vol. 121, no. 11, pp. 959-964, 2010. 
[23] X.-x. Li and D. m. Zhao, "Optical color image encryption with redefined fractional Hartley transform," Optik, vol. 121, no. 7, pp. 673-677, 2010.

[24] Z. j. Liu, L. Xu, T. Liu et al., "Color image encryption by using Arnold transform and color-blend operation in discrete cosine transform domains," Optics Communications, vol. 284, no. 1, pp. 123-128, 2011.

[25] B. Javidi, Optical and Digital Techniques for Information Security, Springer, New York, NY, USA, 2005.

[26] K. Singh, G. Unnikrishnan, and N. K. Nishchal, "Photorefractive optical processing for data security," in Photorefractive Fiber and Crystal Devices: Materials, Optical Properties, and Applications VII, vol. 4803 of Proceedings of SPIE, pp. 205-219, July 2002.

[27] A. Kumar, M. Singh, and K. Singh, "Speckle coding for optical and digital data security applications," in Advances in Speckle Metrology and Related Techniques, G. H. Kaufmann, Ed., WileyVCH, Weinheim, Germany, 2011.

[28] N. r. Zhou, Y. Wang, and L. Gong, "Novel optical image encryption scheme based on fractional Mellin transform," Optics Communications, vol. 284, no. 13, pp. 3234-3242, 2011.

[29] N. r. Zhou, Y. Wang, L. Gong, X. Chen, and Y. Yang, "Novel color image encryption algorithm based on the reality preserving fractional Mellin transform," Optics and Laser Technology, vol. 44, pp. 2270-2281, 2012.

[30] N.-r. Zhou, X. Liu, Y. Zhang, and Y. Yang, "Image encryption scheme based on fractional Mellin transform and phase retrieval technique in fractional Fourier domain," Optics \& Laser Technology, vol. 47, pp. 341-346, 2013.

[31] W. Qin and X. Peng, "Vulnerability to known-plaintext attack of optical encryption schemes based on two fractional Fourier transform order keys and double random phase keys," Journal of Optics A, vol. 11, no. 7, Article ID 075402, 2009.

[32] J. R. Fienup, "Phase retrieval algorithms: a comparison," Applied Optics, vol. 21, no. 15, pp. 2758-2769, 1982.

[33] J. R. Fienup, "Phase retrieval algorithms: a personal tour," Applied Optics, vol. 52, pp. 45-56, 2013.

[34] M. V. Berry, M. R. Jeffrey, and M. Mansuripur, "Orbital and spin angular momentum in conical diffraction," Journal of Optics A: Pure and Applied Optics, vol. 7, no. 11, pp. 685-690, 2005.

[35] M. V. Berry, "Conical diffraction: Hamilton's diabolical point at the heart of crystal optics," in Progress in Optics, E. Wolf, Ed., vol. 50, chapter 2, pp. 13-50, 2007.

[36] J. Garcia, D. Mas, and R. G. Dorsch, "Fractional-Fouriertransform calculation through the fast-Fourier-transform algorithm," Applied Optics, vol. 35, no. 35, pp. 7013-7018, 1996. 

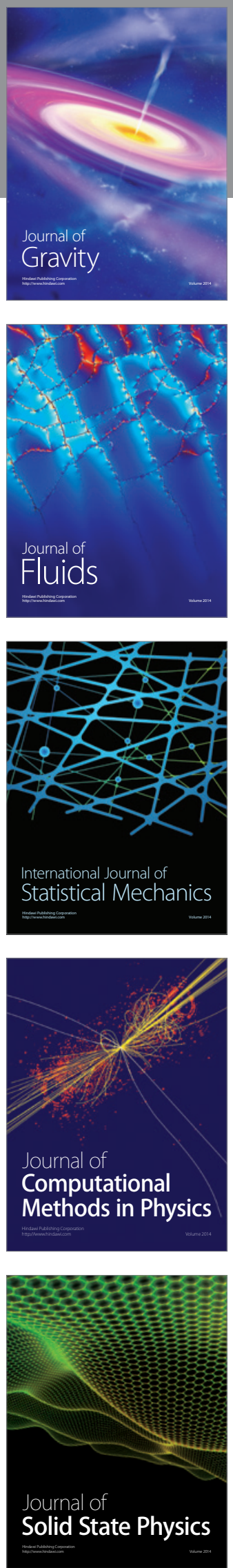

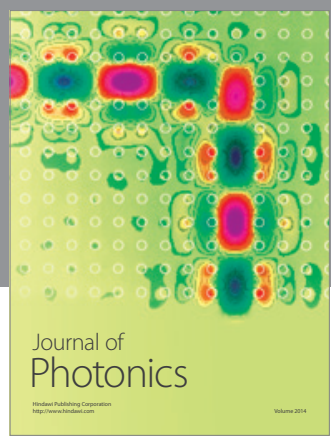

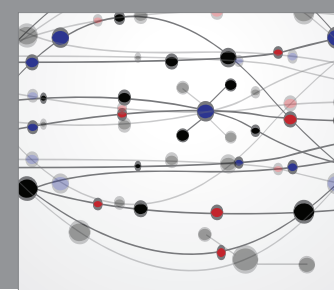

The Scientific World Journal

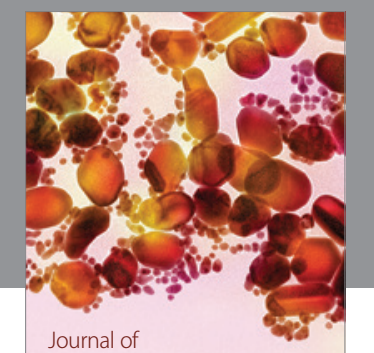

Soft Matter
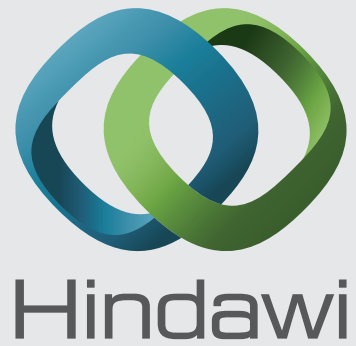

Submit your manuscripts at

http://www.hindawi.com
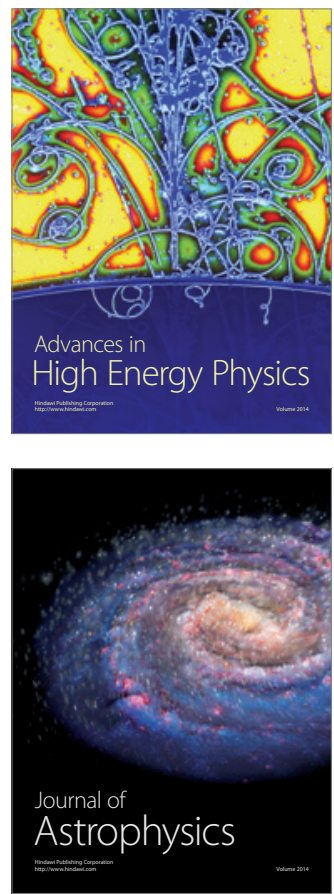
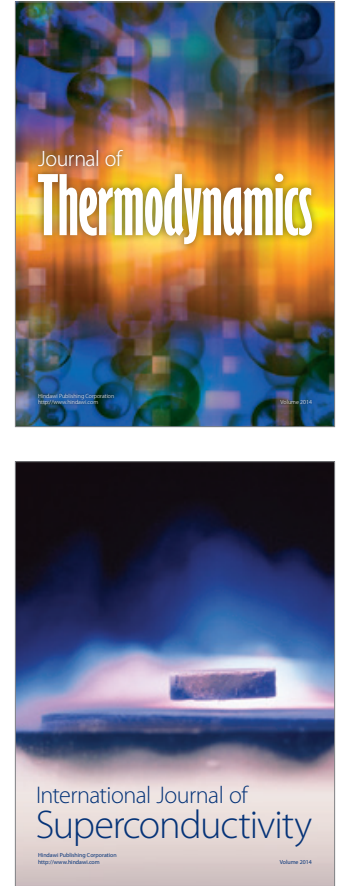
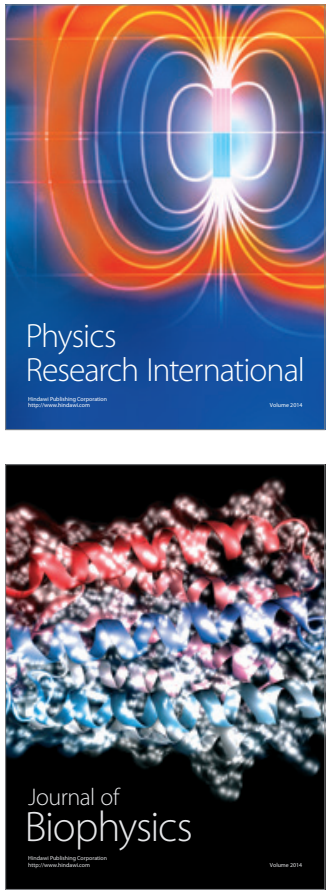
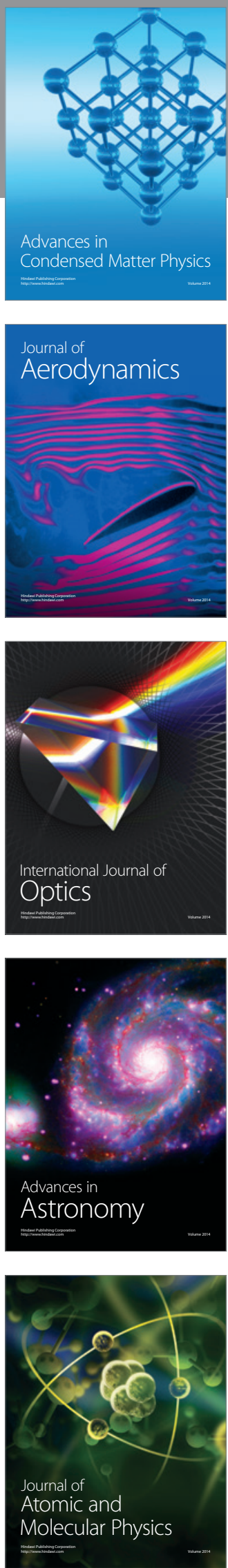